\title{
Optical Gas Sensor Based on the Fiber Laser Transient Regime for Measuring Acetylene
}

\author{
Ana Dinora Guzman-Chavez* and Everardo Vargas-Rodriguez \\ Department of Multidisciplinary Studies, Division of Engineering, Guanajuato University, \\ Av. Universidad s/n, Col Yacatitas, ZIP 38940, Yuriria, Gto., Mexico
}

(Received August 27, 2018; accepted October 31, 2018)

Keywords: gas sensor, acetylene, fiber laser, transient regime, intracavity absorption

An optical gas sensor based on an erbium-doped fiber laser (EDFL) for measuring acetylene $\left(\mathrm{C}_{2} \mathrm{H}_{2}\right)$ is presented. Here, by taking advantage of the thermooptic properties of a silicon ( $\left.\mathrm{Si}\right)$ wafer, the fiber laser emission line is set at $1532.81 \mathrm{~nm}$, where a strong ro-vibrational absorption of the acetylene molecule occurs. Moreover, in our setup, the gas concentration induces cavity losses that affect laser transient characteristics. Here, by measuring the time delay $\left(\tau_{d}\right)$ from the laser transient, it was possible to measure the $\mathrm{C}_{2} \mathrm{H}_{2}$ concentration within the range from 0 to $22 \%$ with a sensitivity of $1.17 \times 10^{-2} \mathrm{~ms} / \%$ and a minimum detectable concentration of 942 ppm. Finally, it is shown that the sensor requires only $18.5 \mathrm{~ms}$ to complete a measurement.

\section{Introduction}

The detection of gases is very important for environmental and industrial purposes; therefore, it is important to have sensors capable of measuring gas concentrations with high precision. For instance, for coal mine hazard detection applications, $\mathrm{CH}_{4}$ sensors with a dynamic range of $0-100 \%{ }^{(1,2)}$ are of interest as well as sensors for monitoring $\mathrm{CO}, \mathrm{O}_{2}$, acetylene $\left(\mathrm{C}_{2} \mathrm{H}_{2}\right)$, and $\mathrm{CO}_{2}{ }^{(1)}$ are required. Therefore, sensors capable of detecting the concentration of each of these gases with high sensitivity and low cross sensitivity to other gases are necessary. In particular, $\mathrm{C}_{2} \mathrm{H}_{2}$ is a highly flammable gas that is used in several industrial applications. Its lower explosive limit of $2.5 \%$ and upper explosive limit of $100 \%$ by volume make this gas extremely hazardous. Currently, a broad range of acetylene sensors based on different technologies, such as metal oxide semiconductor (MOS), ${ }^{(3-5)}$ optical, ${ }^{(6-8)}$ and electrochemical sensors, ${ }^{(9)}$ are available. Each of these technologies has advantages and limitations. For instance, MOS sensors are small and can be integrated on a chip; however, their sensing element needs to be operated at a high temperature $\left(<150^{\circ} \mathrm{C}\right)$. Moreover, their response time $\left(\tau_{s}\right)$ and recovery time $\left(\tau_{r}\right)$ are on the order of seconds and some of them have poor selectivity. For instance, Iftekhar Uddin et al. ${ }^{(3)}$ developed a Ag-loaded $\mathrm{ZnO}-\mathrm{Gr}$ hybrid nanostructure-based acetylene gas sensor with a measurement concentration range of 1-10000 ppm, a $\tau_{s} / \tau_{r}$ of 25/80 $\mathrm{s}$, and an operating temperature of $150{ }^{\circ} \mathrm{C}$. Tamaekong et al. reported an acetylene sensor based

*Corresponding author: e-mail: ad.guzman@ugto.mx https://doi.org/10.18494/SAM.2018.2114 
on $\mathrm{Pt} / \mathrm{ZnO}$ thick films with a measurement concentration range of 836-10000 ppm, a $\tau_{s} / \tau_{r}$ of $6 / 65 \mathrm{~s}$, and an operating temperature of $300{ }^{\circ} \mathrm{C} .{ }^{(4)}$ Qiao et al. reported an acetylene sensor based on mesoporous $\mathrm{ZnO}$ nanosheets that achieved a measurement concentration range of 1-4000 ppm, with a $\tau_{s} / \tau_{r}$ of $11 / 5 \mathrm{~s}$, and it was operated at $400{ }^{\circ} \mathrm{C} .{ }^{(5)}$ Moreover, some MOS-based sensors can have limited selectivity; therefore, their response can be affected by other molecules, such as water vapor. On the other hand, optical sensors are another important option to detect gases and there exist different architectures to implement them. Typically, these gas sensors have a faster response and can have a high sensitivity and a high selectivity to the target molecule. However, these sensors can be more expensive and have complex technical configurations. In particular, gas sensors based on erbium-doped fiber lasers (GS-EDFLs) can be used to monitor different gases that have absorption bands within the NIR region, since the gain of erbium-doped fiber (EDF) amplifiers covers the C and L telecommunication bands. ${ }^{(10)}$ The another advantage that EDF systems offer is that these can be implemented with commercial fiber components allowing the use of cost-effective GS-EDFLs. Besides, some erbium-doped fiber laser (EDFL) configurations offer frequency stability and fine tuning capabilities, ${ }^{(11-13)}$ which are important characteristics for designing gas sensors based on optical spectroscopy techniques. For instance, some authors have presented GS-EDFLs based on the direct absorption tunable laser absorption spectroscopy (DA-TLAS) technique. In this technique, the laser emission line is tuned around the spectral region where one or some ro-vibrational absorption lines of the target gas occur in order to resolve, analyze, and then determine its concentration. ${ }^{(6-8)}$ Therefore, this type of sensor requires a laser whose emission can be spectrally tuned and free of mode hopping over the spectral range where an absorption line of the target gas occurs. Additionally, some authors have proposed GS-EDFL based on the linelocked tunable laser absorption spectroscopy (LL-TLAS) technique, where laser emission must be wavelength-locked instead of being tuned. ${ }^{(14-16)}$ Here, the laser emission wavelength must be matched with one absorption line peak of the molecule, which implies that the laser must have very high wavelength selectivity and stability. In the mentioned works based on these two last techniques, the gas cell can be either inside ${ }^{(8,14-16)}$ or outside $e^{(6,7)}$ of the laser cavity. Basically, in ideal sensors based on TLAS, the laser intensity must remain quasi constant and is monitored by an optical detector after the gas cell. Here, the changes in laser intensity will be due to the level of absorption of the ro-vibrational line, which will directly depend on the concentration.

Furthermore, in other GS-EDFL sensors, the gas is used to induce losses in the laser cavity, in order to induce changes in laser transient behavior. ${ }^{(17-20)}$ These configurations make it possible to acquire the sensor output signal easily and accurately using reliable and low-cost electronics, and to process this information to determine the gas concentration. In this type of sensor, the laser pump power is modulated in order to allow the measurement of the laser transient behavior. Therefore, in this work, a gas sensor based on an erbium-doped fiber ring laser for measuring $\mathrm{C}_{2} \mathrm{H}_{2}$ is presented. Here, the EDFL is based on a silicon ( $\mathrm{Si}$ ) wafer that acts as a spectrally tunable filter. The spectrum generated by the $\mathrm{Si}$ wafer can be finely shifted and locked by controlling the temperature of the wafer. The temperature was adjusted in order to match the EDFL center wavelength with the center of a strong ro-vibrational absorption line of acetylene. Here, the gas cell was incorporated in the cavity ring laser and therefore cavity 
losses were varied depending on the $\mathrm{C}_{2} \mathrm{H}_{2}$ concentration $(C)$. These cavity losses affect the time delay $\left(\tau_{d}\right)$, which is taken as the reference characteristic of the laser transient. Hence, we were able to determine $C$ by monitoring $\tau_{d}$ with a maximum sensitivity of $1.17 \times 10^{-2} \mathrm{~ms} / \%$ for the concentration range of $0-22 \%$ and a minimum detectable level of $942 \mathrm{ppm}$. Finally, it is demonstrated that one main advantage of this sensor arrangement is that it can be used for quasi real-time applications since its response time is of $18.5 \mathrm{~ms}$.

\section{Experimental Setup}

The sensor arrangement based on an EDFL for measuring acetylene is shown in Fig. 1. Here, a piece of EDF (Liekki Er80-8/125) of $98 \mathrm{~cm}$ length was pumped with a pigtailed diode laser, emitting at $\lambda=976 \mathrm{~nm}$ and delivering a maximum output power of $300 \mathrm{~mW}$, through a wavelength division multiplexer (WDM). The luminescence generated by the EDF traveled from ports 1 to 2 of the circulator where the Si wafer of $85 \mu \mathrm{m}$ thickness was coupled. A thermoelectric cooler (TEC) was used to vary the Si wafer temperature. The interference spectrum generated by the wafer was used to define the laser emission wavelength. This spectrum was reflected towards port 3 of the circulator and, afterwards, it was divided into two signals with the 90/10 coupler. Ten percent of the signal was recorded using an optical spectrum analyzer (OSA, Yokowaga AQ6370C) with a resolution of $0.02 \mathrm{~nm}$ and used to monitor the laser emission wavelength. The $90 \%$ output was spliced to a pigtailed collimator that was assembled by using a GRIN lens, a matching sleeve, and a pigtailed ferrule. This collimator was aligned with a microscope objective and an $\mathrm{FC} / \mathrm{PC}$ fiber optic pigtail that was spliced to a 50/50 coupler. Between these components, the gas cell was placed, where the gas-light interaction occurred. One output of the 50/50 coupler was spliced to a variable optical attenuator (VOA) and afterwards to the WDM in order to close the ring cavity. The laser output was provided by the signal of the other output of the 50/50 coupler, which was monitored with a photodetector (PD) and its waveform was recorded with an oscilloscope.

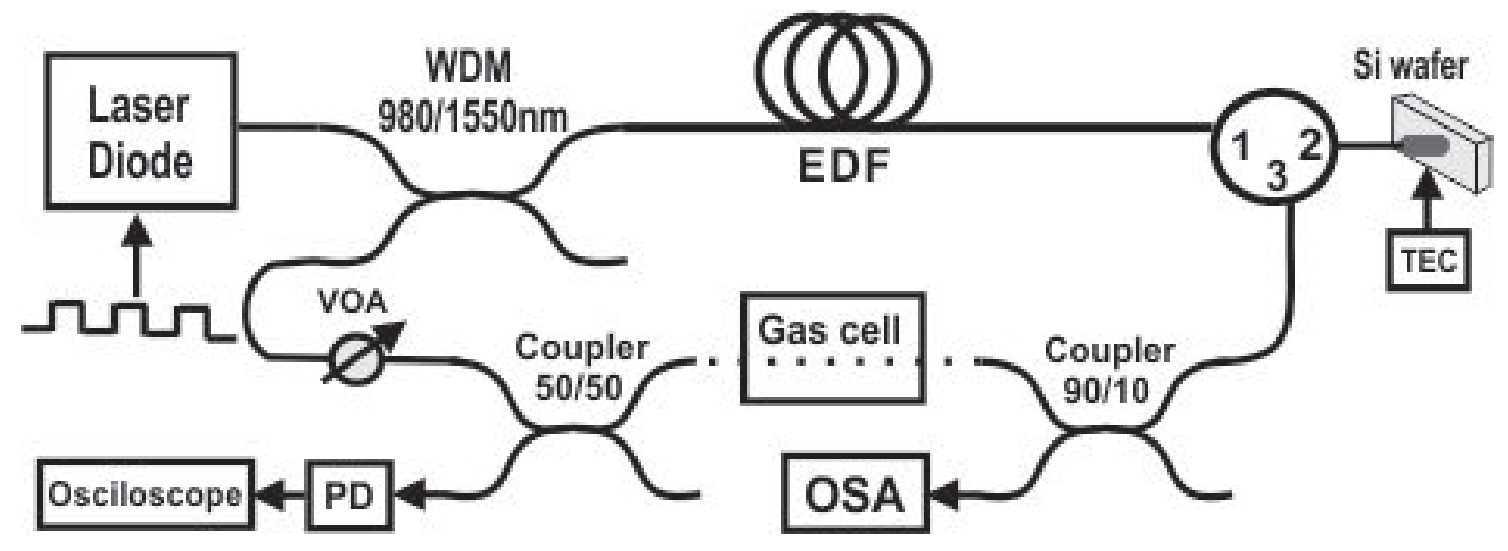

Fig. 1. Experimental setup for measuring $\mathrm{C}_{2} \mathrm{H}_{2}$ concentration. 


\subsection{Operation principle and measurement technique}

In this work, the transient behavior of the EDFL based on a Si wafer was investigated as the $\mathrm{C}_{2} \mathrm{H}_{2}$ concentration was varied. It is known that the laser transient basically depends on medium properties, pump power, and cavity losses. ${ }^{(21)}$ In our setup, cavity losses can be modified by the interaction of light with the target gas as the gas cell is part of the laser cavity (Fig. 1). For such a reason, it is necessary that the emission line of the EDFL is centered at the wavelength where one strong absorption of the gas molecule occurs in order to maximize cavity losses. The EDFL used for sensing gas absorption was based on a bulk, uncoated, and double-side-polished Si wafer of $85 \mu \mathrm{m}$ thickness, which acts as a spectrally tunable FabryPerot filter. By taking advantage of the thermooptic properties of $\mathrm{Si}$, the interference spectrum generated by the wafer could be finely shifted over a spectral window of $\sim 3.9 \mathrm{~nm}$ width, which is indeed the free spectral range (FSR) of such a spectrum. Hence, by varying the temperature of the wafer, we can finely tune the emission line of our EDFL. The wafer temperature was set with a standard TEC, which was stabilized with a proportional-integral-derivative controller electronic stage. This allowed us to maintain the temperature of the Si wafer highly stable, with a standard deviation of $0.02{ }^{\circ} \mathrm{C}$. This shows that the laser emission wavelength is fixed with an estimated standard deviation of approximately $0.12 \mathrm{pm}$. To use our EDFL to sense $\mathrm{C}_{2} \mathrm{H}_{2}$, the emission line of the EDFL was set at $1532.81 \mathrm{~nm}$, where a strong ro-vibrational absorption of the acetylene molecule occurred (Fig. 2). It is important to mention that the VOA (Fig. 1) was used to induce additional cavity losses and adjust the laser operation and performance.

Once the mechanism to induce cavity losses was set, the pump power was modulated with a pulse train waveform $\left(M_{S}\right)$. In this manner, it is possible to monitor the laser transient behavior when the laser pump is suddenly switched on and until it becomes stable over time. In particular, from the laser transient behavior parameters, we focused on the time delay $\left(\tau_{d}\right)$. It is defined as the time between the moment when the pump is switched on and until laser emission $\left(L_{S}\right)$ reaches the midamplitude $\left(A_{L S} / 2\right)$ of the level when $L_{S}$ is stabilized [see Fig. 3(a)].

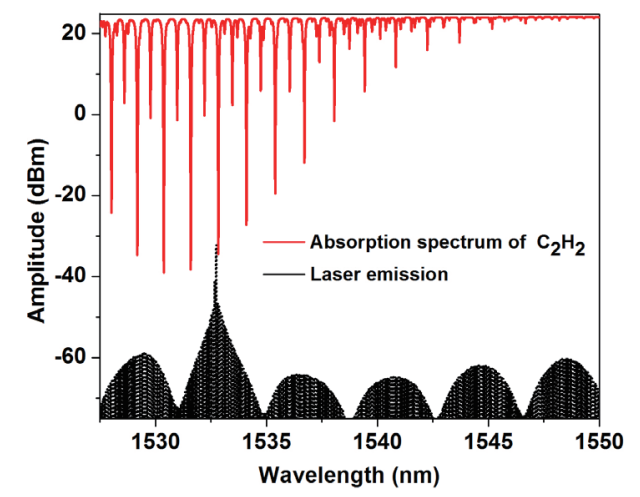

Fig. 2. (Color online) Absorption spectrum of $\mathrm{C}_{2} \mathrm{H}_{2}$ and laser emission of EDFL based on a Si wafer. The $\mathrm{C}_{2} \mathrm{H}_{2}$ spectrum is shifted to $22 \mathrm{dBm}$ for clarity. 


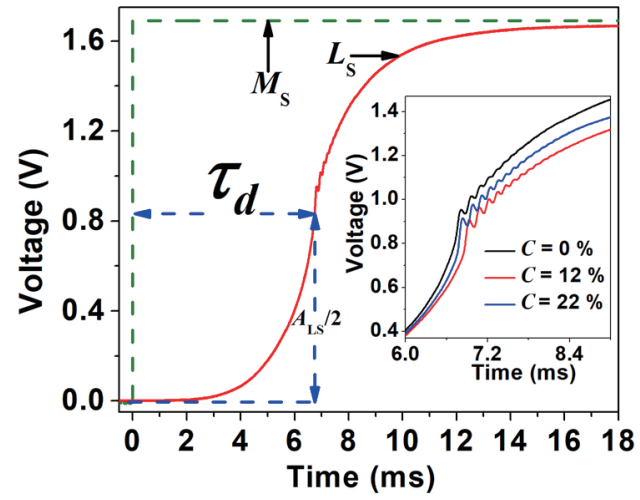

(a)

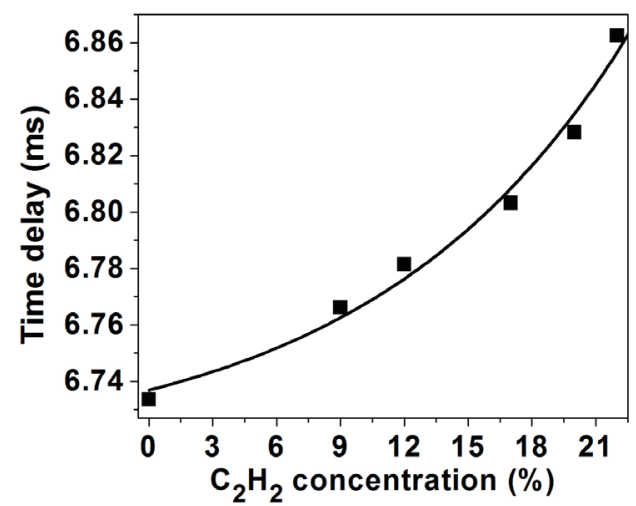

(b)

Fig. 3. (Color online) (a) Laser transient behavior $\left(L_{S}\right)$ as the pump diode laser is modulated with the pulse waveform $M_{S}$ and definition of $\tau_{d}$; in the inset of (a), the laser transient $L_{S}$ for different concentrations is shown. (b) Laser time delay as a function of $\mathrm{C}_{2} \mathrm{H}_{2}$ concentration measured for the concentration range from 0 to $22 \%$.

In our experimental work, the laser transient behavior was measured using a fast photodiode (PD) and recorded using an oscilloscope. In addition, the frequency of $M_{S}$ will be limited by the time that the laser requires to reach the stabilization level $A_{L S}\left(t_{o n}\right)$ plus the time required by the laser to reach the off state $\left(t_{o f f}\right)$. Therefore, the maximum modulation frequency, expressed as $f_{\max }=1 /\left(t_{o n}+t_{o f f}\right)$, will be limited by $t_{\text {on }}$ and $t_{\text {off }}$, which are obtained when the laser reaches the lower $\tau_{d}$.

\section{Results and Discussion}

In our experiment, the pump power was modulated with $M_{S}$ at $20 \mathrm{~Hz}$ in such a way that it emitted 0 and $243 \mathrm{~mW}$ for the low and high levels, respectively [Fig. 3(a)]. Moreover, by adjusting the VOA and TEC temperature, the laser was tuned to $1532.81 \mathrm{~nm}$ with the cell filled with $0 \% \mathrm{C}_{2} \mathrm{H}_{2}$ concentration. At this concentration, $\tau_{d}$ is related to the static cavity losses of the EDFL. Afterwards, according to the Beer-Lambert law, ${ }^{(2)}$ the cavity losses were varied by changing the $\mathrm{C}_{2} \mathrm{H}_{2}$ concentration. Here, the time required to reach the laser threshold condition will increase as the cavity losses increase. ${ }^{(21,23)}$ In the inset of Fig. 3(a), we observed that the time delay becomes longer as the $\mathrm{C}_{2} \mathrm{H}_{2}$ concentration increases. In Fig. 3(b), a detailed experimental evolution of the time delay as a function of $\mathrm{C}_{2} \mathrm{H}_{2}$ concentration and its exponential fit are presented. Here, the $\mathrm{C}_{2} \mathrm{H}_{2}$ concentration range was from 0 to $22 \%$, and the high level of pump power was $243 \mathrm{~mW}$. From this figure, the nonlinear behavior of $\tau_{d}$ becomes evident as $C$ increases. This behavior is due to the nonlinearity of the laser dynamics itself and the nonlinear increase in losses as a function of gas concentration. In addition, for the pump power with a high level of $243 \mathrm{~mW}, t_{\text {on }}$ was $18 \mathrm{~ms}$ and $t_{\text {off }}$ was $0.5 \mathrm{~ms}$ when $C$ was $0 \%$, allowing $M_{S}$ to be modulated with a $f_{\max }$ of $54 \mathrm{~Hz}$. 


\subsection{Sensitivity and minimum detectable concentration}

The laser transient behavior depends on several parameters and, in general, the time delay $\left(\tau_{d}\right)$ will be reduced as the power increases because a shorter time is required to reach the threshold population inversion as the gain of the EDF increases. ${ }^{(21,23)}$ Therefore, to observe the effects over the laser transient, the pump diode laser was modulated with three different train pulse waveforms, in which their high levels were 243, 250, and $257 \mathrm{~mW}$ and their modulation frequency was $20 \mathrm{~Hz}$. For these power levels, the measured $\tau_{d}$ as a function of $C$ is presented in Fig. 4. Here, it can be observed that $\tau_{d}$ decreases as the power pumped by the diode increases, as it was expected.

In addition, the sensor sensitivity can be expressed as the ratio between the time delay change and the $\mathrm{C}_{2} \mathrm{H}_{2}$ concentration change, $S=\Delta \tau_{d} / \Delta C$. Here, the fitting curves of the experimental results shown in Fig. 4 were used to estimate the sensor sensitivities, which are shown in Fig. 5. From this figure, we noted that $\tau_{d}$ becomes less sensitive to changes in gas concentration as the pump power increases. This is because the laser transient characteristics are more sensitive to changes in given laser parameter (in our case, cavity losses) when the pump power is just above the laser threshold. ${ }^{(20,23-25)}$ Consequently, the sensor sensitivity will be governed by the compromise between the power delivered by the diode laser and the cavity losses. In this way, for our sensing arrangement, the longer $S$ was approximately $1.17 \times 10^{2}$ $\mathrm{ms} / \%$ for the concentration range of 0 to $22 \%$ when the pump diode laser delivered pulses of $243 \mathrm{~mW}$ (Fig. 5).

The minimum detectable concentration was estimated as $C$ for which the signal-to-noise ratio is equal to 1 , which can be expressed as $\left[\tau_{d}(C)-\tau_{d}\left(C_{0}\right)\right] / \sigma=1$, where $\sigma$ is the standard deviation of the time delay when the $\mathrm{C}_{2} \mathrm{H}_{2}$ concentration is $C_{0}=0 \%$ and $\tau_{d}(C)$ is the fitted time delay as a function of the concentration (Fig. 4). Therefore, from a series of time delay transient

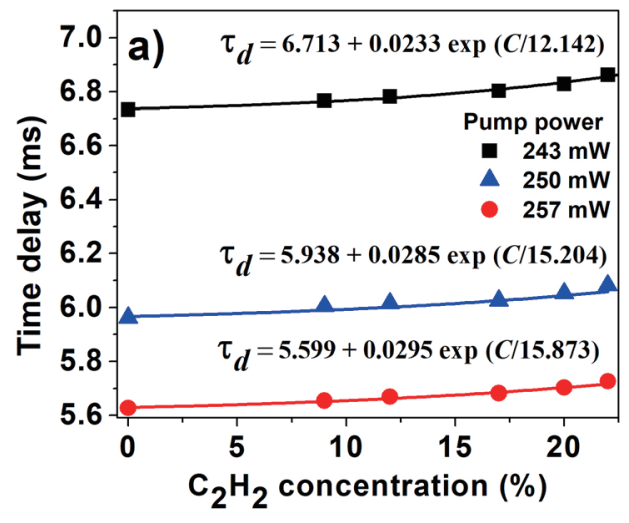

Fig. 4. (Color online) Time delay as a function of $\mathrm{C}_{2} \mathrm{H}_{2}$ concentration considering that the pump diode is modulated with a pulse train waveform $\left(M_{S}\right)$ with three different power levels.

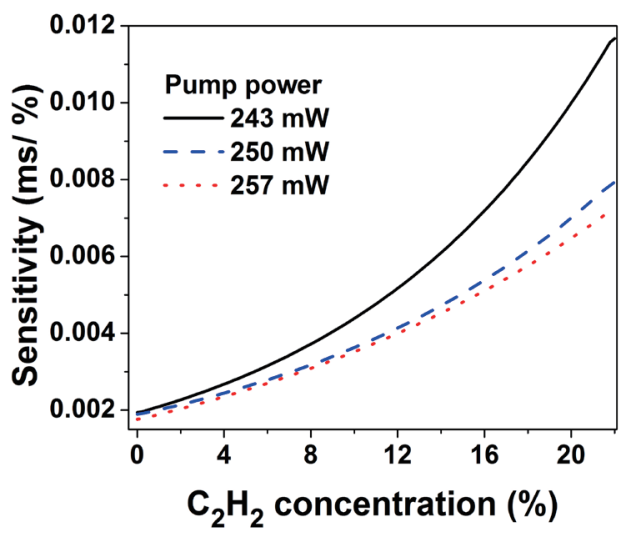

Fig. 5. (Color online) Sensitivity as a function of $\mathrm{C}_{2} \mathrm{H}_{2}$ concentration. 
graphs measured during $5 \mathrm{~min}$, a $\sigma$ of $1.8 \times 10^{-4} \mathrm{~ms}$ was obtained. In this manner, the estimated minimum detectable concentration was $942 \mathrm{ppm}$ when the pump diode laser delivered pulses of $243 \mathrm{~mW}$.

\section{Conclusions}

In this work, we reported an acetylene gas sensor in which a gas cell is inserted into the cavity of an EDFL and is based on a silicon wafer. By taking advantage of the thermooptic properties of silicon, it was possible to center the laser emission where a strong ro-vibrational absorption line of the acetylene molecule occurs. Here, the acetylene concentration was determined from the time delay characteristics of the laser transient response. In this manner, the sensitivity achieved for the concentration range of $0-22 \%$ was approximately $1.17 \times$ $10^{-2} \mathrm{~ms} / \%$ and the minimum detectable concentration was $942 \mathrm{ppm}$. Finally, this sensor arrangement can be used for quasi real-time applications since it can be used to measure the gas concentration in $18.5 \mathrm{~ms}$.

\section{Acknowledgments}

This work was supported by the Universidad de Guanajuato.

\section{References}

1 T. Liu, Y. Wei, G. Song, B. Hu, L. Li, G. Jin, J. Wang, Y. Li, C. Song, Z. Shi, L. Zhao, J. Hu, W. Zhao, M. Hou, R. Li, and J. Wang: Measurement 124 (2018) 211. https://doi.org/10.1016/j.measurement.2018.03.046

2 A. Zakrzewski and S. Patela: Sens. Actuators, A 256 (2017) 51. https://doi.org/10.1016/j.sna.2017.01.019

3 A. S. M. Iftekhar Uddin, D.-T. Phan, and G.-S. Chung: Sens. Actuators, B 207 (2015) 362. https://doi. org/10.1016/j.snb.2014.10.091

4 N. Tamaekong, C. Liewhiran, A. Wisitsoraat, and S. Phanichphant: Sens. Actuators, B 152 (2011) 155. https:// doi.org/10.1016/j.snb.2010.11.058

5 P.-Y. Qiao, L.-X. Zhang, M.-Y. Zhu, Y.-Y. Yin, Z.-W. Zhao, H.-N. Sun, J.-Y. Dong, and L.-J. Bie: Sens. Actuators, B 250 (2017) 189. https://doi.org/10.1016/j.snb.2017.04.158

6 J. Wang and H. Wang: Infrared Phys. Technol. 65 (2014) 1. https://doi.org/10.1016/j.infrared.2014.03.002

7 S. Zheng, Y. Zhu, and S. Krishnaswamy: SPIE Smart Structures and Materials + Nondestructive Evaluation and Health Monitoring 8692 (2013) 9. https://doi.org/10.1117/12.2001325

8 H. Zhang, Y. Lu, L. Duan, Z. Zhao, W. Shi, and J. Yao: Opt. Express 22 (2014) 24545. https://doi.org/10.1364/ OE.22.024545

9 C. Li, Y. Su, X. Lv, H. Xia, and Y. Wang: Sens. Actuators, B 149 (2010) 427. https://doi.org/10.1016/ j.snb.2010.05.011

10 S. Yamashita: J. Sel. Top. Quantum Electron. 7 (2001) 41. https://doi.org/10.1109/2944.924007

11 E. Gallegos-Arellano, E. Vargas-Rodriguez, A. D. Guzman-Chavez, M. Cano-Contreras, J. L. Cruz, and R. K. Raja-Ibrahim: Laser Phys. Lett. 13 (2016) 065102. https://doi.org/10.1088/1612-2011/13/6/065102

12 J. Marshall, G. Stewart, and S. Whitenett: Meas. Sci. Technol. 17 (2006) 1023. https://doi.org/10.1088/0957$0233 / 17 / 5 / \mathrm{S} 15$

13 G. Whitenett, G. Stewart, H. Yu, and B. Culshaw: J. Lightwave Technol. 22 (2004) 813. https://doi.org/10.1109/ JLT.2004.824530

14 R. Romero, F. M. Araújo, and L. A. Ferreira: Appl. Opt. 46 (2007) 7900. https://doi.org/10.1364/AO.46.007900

15 Y. Zhang, M. Zhang, W. Jin, H. L. Ho, M. S. Demokan, B. Culshaw, and G. Stewart: Opt. Commun. 232 (2004) 295. https://doi.org/10.1016/j.optcom.2004.01.013

16 Y. Zhang, M. Zhang, and W. Jin: Opt. Commun. 220 (2003) 361. https://doi.org/10.1016/S0030-4018(03)014214 
17 G. Stewart, G. Whitenett, and B. Culshaw: Optics East 20066371 (2006) 11. https://doi.org/10.1117/12.684669

18 S. George, S. Peter, and C. Brian: Meas. Sci. Technol. 15 (2004) 1621. https://doi.org/10.1088/09570233/15/8/033

19 H. Arellano-Sotelo, A. V. Kir’yanov, Y. O. Barmenkov, and V. Aboites: Opt. Laser Technol. 43 (2011) 132. https://doi.org/10.1016/j.optlastec.2010.05.017

20 Y. O. Barmenkov, A. Ortigosa-Blanch, A. Diez, J. L. Cruz, and M. V. Andrés: Opt. Lett. 29 (2004) 2461. https://doi.org/10.1364/OL.29.002461

21 G. Stewart, G. Whitenett, K. Vijayraghavan, and S. Sridaran: J. Lightwave Technol. 25 (2007) 1786. https:// doi.org/10.1109/JLT.2007.899192

22 R. Horne: Particulate Emissions - Optical and Other Methods for Continuous Monitoring from a Point Source In Industrial Air Pollution Monitoring (Chapman \& Hall, New York, 1998) Chap. 4. https://doi. org/10.1007/978-94-009-1435-3

23 H. K. Hisham, G. A. Mahdiraji, A. F. Abas, M. A. Mahdi, and F. R. M. Adikan: IEEE Photonics J. 4 (2012) 2353. https://doi.org/10.1109/JPHOT.2012.2231957

24 Y. Zhang, M. Zhang, and W. Jin: Sens. Actuators, A 104 (2003) 183. https://doi.org/10.1016/S09244247(03)00058-X

25 A. D. Guzmán-Chávez, A. Díez, J. L. Cruz, and M. V. Andrés: Laser Phys. 22 (2012) 579. https://doi. org/10.1134/S1054660X12030061

\section{About the Authors}

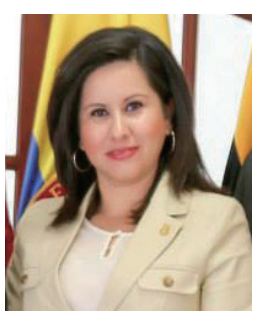

Ana Dinora Guzman-Chavez received her M.S. and Ph.D. degrees from the Research Center of Optics, Mexico, in 2007 and 2010, respectively. In 2011, she did a postdoctoral studies at the University of Valencia, Spain. Since 2012, she has been a professor at Guanajuato University. Her research interests are in optical sensors, fiber lasers, and interferometry.

(ad.guzman@ugto.mx)

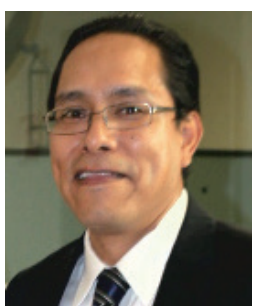

Everardo Vargas-Rodriguez completed his M.Sc. in instrumentation at the University of Guanajuato, Mexico. Later, he received a Ph.D. in Optoelectronics at the University of Southampton in 2007. He joined the University of Guanajuato in Mexico in 2007 as a professor. He served as a Director of the Department for Multidisciplinary Studies from 2008-2016. His research interest involves optoelectronics sensors, interferometry, and fiber lasers. (evr@ugto.mx) 